\title{
Effects of Microhole Sidewall Confinement on Bubble Growth and Bubble-Generated Shock Waves
}

\author{
${ }^{a}$ Navid Dabir-Moghaddam and ${ }^{b}$ Benxin Wu \\ ${ }^{a}$ Illinois Institute of Technology, Chicago, IL \\ ${ }^{\mathrm{b}}$ Corresponding author: Associate Professor, School of Mechanical Engineering, Purdue University, 585 \\ Purdue Mall, West Lafayette, IN 47907, email: wu65@ purdue.edu; Adjunct Associate Professor, Illinois \\ Institute of Technology, Chicago, IL 60616, email: bwu11@iit.edu.
}

\begin{abstract}
The evolution of bubbles in water may be a critical process in many technologies or applications, including several manufacturing processes. Despite the previous work on cavitations and bubbles, the prior investigations in the literature are not sufficient about the effect of a micro-scale structure (such as a microhole sidewall) confinement on the bubble evolution and on the bubble-generated shock waves. In this paper, this effect has been studied using a physics-based model, which has been verified by comparing its predictions with experimental measurements in the literature on bubble evolutions in water without a micro-scale structure confinement. Under the investigated conditions, it has been found that due to the reflection of the shock waves by the microhole sidewall and the interactions among the bubble and the reflected waves, the peak pressure on the hole bottom wall surface has been significantly enhanced. It is good work in the future to study the implications of the discovery on related manufacturing processes and other applications, and how to intentionally utilize the pressure enhancement effect to benefit the related applications and manufacturing processes.
\end{abstract}

Keywords: cavitations and bubbles

\section{Introduction}

The evolution of bubbles in water or other liquids may be a critical process in many technologies or applications (including several manufacturing processes), such as ultrasonic cleaning, cavitation peening, laser-material interactions in liquids, and medical treatment processes based on ultrasound [1-5]. A bubble, when it collapses or shrinks to a very small size, may have a very high pressure $[3,5]$. The high-pressure bubble may re-grow and send a shock wave into its surrounding liquid medium. If the spatial confinement of a nearby microscale structure (such as the sidewall of a microhole) exists, the bubble growth and the bubblegenerated shock wave may be significantly affected. A good understanding of the effect of the 
microscale structure confinement on the bubble growth and the bubble-generated shock wave may provide valuable information to related applications, and help process designs and technology improvements.

For example, a cavitation peening process utilizes the shock wave generated during bubble evolutions to impact a workpiece surface to induce surface compressive residual stresses, which is to enhance the workpiece mechanical properties (e.g., fatigue properties) [1]. If a cavitation peening process is used to process a workpiece with surface micro features (e.g., microholes), then bubble growth in a microhole may happen and play a very critical role in the manufacturing process.

However, the reported investigations are not sufficient about the microscale structure confinement effect on the bubble growth and the bubble-generated shock wave, despite the previous studies in the literature on cavitations and bubbles (e.g., [3-12]).

In this paper, the microhole sidewall confinement effect will be studied using a physicsbased model, which is developed by numerically solving the two-dimensional (2D) axisymmetric compressible fluid mechanics equations, and can simulate the evolution of a bubble in water. First, the model will be verified by comparing its predictions with experimental measurements in the literature for bubble evolutions in water near a solid surface without a microscale structure confinement. Then the experimentally tested model will be applied to study the effect of a microhole sidewall on the growth of a high-pressure small bubble inside the hole and the effect on the bubble-generated shock wave. It will be shown later in this paper that due to the confinement effect of the microhole sidewall, the peak pressure induced on the flat solid surface below the bubble (that is, the hole bottom wall surface) has been significantly enhanced, and the 
enhancement is due to the reflection of shock waves by the sidewall and the interactions among the bubble and the reflected waves.

\section{Model}

Figure 1 shows the schematic diagram for the model based on a $2 \mathrm{D}$ axisymmetric domain, where $r$ and $z$ are spatial coordinates. It is assumed that a bubble pre-exists at $\mathrm{t}=0$ and is surrounded by a liquid water medium. The bubble center is on the symmetric axis. It is assumed that the pressure at the two boundaries of the water medium at $r=r_{\max }$ and $\mathrm{z}=\mathrm{z}_{\max }$ is equal to the ambient pressure of 1 bar. For the simulation results reported in this paper, the values of $r_{\max }$ and $z_{\max }$ are chosen to be sufficiently large such that the shock wave due to bubble evolutions does not reach the boundaries of $\mathrm{z}=\mathrm{z}_{\max }$ or $\mathrm{r}=\mathrm{r}_{\max }$ during the simulated period. Two situations are simulated in this paper. In Fig.1a, a bubble evolves above a solid and rigid flat surface, and the initial distance between the bubble center and the solid surface is equal to $s$. In Fig.1b, a bubble evolves inside a microhole that has a radius of $R$ and a depth of $H$. The microhole has a solid and rigid bottom wall and sidewall. The comparisons of the simulation results for the two situations can reveal the spatial confinement effect of the microhole sidewall on the bubble evolution and the bubble-generated shock waves. The model is based on 2D axisymmetric fluid mechanics equations supplemented by suitable equations of state (EOS), and the bubble wall (i.e., the liquid-vapor interface) is handled through the volume of fluid (VOF) method, and the surface tension at the bubble wall is handled through the continuum surface force (CSF) model [6-9, 13-25].

The domain boundary conditions and the liquid water initial conditions assumed in the model calculations are given in Fig. 1, while the assumed initial conditions for the bubble (and 
the vapor inside the bubble) are given in Fig. 2 (for the simulation results shown in Fig. 3) and Fig. 4 (for the simulation results in Figs. 5 to 9).

The dynamic evolution of the vapor inside the bubble and the water outside the bubble are governed by the 2D compressible axisymmetric fluid mechanics equations [13-14, 17-19]:

$\frac{\partial \vec{U}}{\partial t}+\frac{1}{r} \frac{\partial \vec{E}}{\partial r}+\frac{\partial \vec{F}}{\partial z}=\vec{S}$

$\vec{U}=\left[\begin{array}{l}\rho \\ \rho v_{r} \\ \rho v_{z} \\ \rho e\end{array}\right], \quad \vec{E}=\left[\begin{array}{l}\rho r v_{r} \\ \rho r v_{r}^{2} \\ \rho r v_{r} v_{z} \\ r v_{r}(\rho e+p)\end{array}\right], \quad \vec{F}=\left[\begin{array}{c}\rho v_{z} \\ \rho v_{r} v_{z} \\ \rho v_{z}^{2} \\ v_{z}(\rho e+p)\end{array}\right]$

$$
\vec{S}=\left[\begin{array}{c}
0 \\
-\frac{\partial p}{\partial r}+\frac{1}{r} \frac{\partial\left(r \tau_{r r}\right)}{\partial r}+\frac{\partial \tau_{z r}}{\partial z}-\frac{\tau_{\theta \theta}}{r}+F_{r} \\
-\frac{\partial p}{\partial z}+\frac{\partial \tau_{z z}}{\partial z}+\frac{1}{r} \frac{\partial\left(r \tau_{r z}\right)}{\partial r}+F_{z} \\
\frac{1}{r} \frac{\partial}{\partial r}\left(r k \frac{\partial T}{\partial r}\right)+\frac{\partial}{\partial z}\left(k \frac{\partial T}{\partial z}\right)+\nabla \cdot(\overline{\bar{\tau}} \cdot \vec{v})+\vec{F} \cdot \vec{v}
\end{array}\right]
$$

where $t$ represents time, $r$ and $z$ denote spatial coordinates, $\rho$ is the density, $\mathrm{v}_{\mathrm{r}}$ and $\mathrm{v}_{\mathrm{z}}$ represent the components of velocity in $\mathrm{r}$ and $\mathrm{z}$ directions, respectively, $\mathrm{p}$ denotes pressure, $\vec{v}$ is the velocity vector, $\overline{\bar{\tau}}$ is the viscous stress tensor, $\tau_{\mathrm{rr}}, \tau_{\mathrm{zr}}, \tau_{\mathrm{rz}}, \tau_{\theta \theta}, \tau_{\mathrm{zz}}$ are viscous stress components, $e$ represents the total energy per unit mass (including internal and kinetic energies), $T$ represents temperature, $k$ denotes thermal conductivity, $\vec{F}$ represents the body force (per unit volume) vector, and $F_{r}$ and $F_{z}$ denote the body force components in $r$ and $z$ directions, respectively. The effect of the gravity force is assumed to be small and is neglected in the model.

It is assumed that water vapor is inside the bubble, for which the ideal gas equation of state (EOS) has been used in the simulation [15]. For the water outside the bubble, the Tait EOS has been applied [7-9]: 


$$
\frac{p+B}{p_{0}+B}=\left(\frac{\rho}{\rho_{0}}\right)^{n}
$$

where $\mathrm{P}_{0}$ and $\rho_{0}$ denote the steady ambient pressure ( 1 bar) and the corresponding water density, respectively, and the parameter $B$ is equal to around 3000 bar and $n$ is around 7 for water [7-9].

The bubble boundary wall (that is, the vapor-liquid interface) is handled through the volume of fluid (VOF) method [6, 14, 16, 20-25]. Surface tension at the bubble boundary wall is handled through the continuum surface force (CSF) model [6, 14, 22-25], which has resulted in the volume force source terms in the governing equations (the $F_{r}$ and $F_{z}$ terms).

It should be noted that the VOF method is a well-established and well-known method to handle the interface between two phases (like the bubble wall in this study, which is a liquidvapor interface). Its detailed introductions and information can be found in $[14,16,20,21]$. The VOF method has been applied by numerous researchers (e.g., the authors of $[6,22,24,25]$ applied the VOF method to study bubble evolutions). The CSF model is a well-known method for handling surface tension, and its detailed introductions and information can be found in [14, 22, 23], and it has also been applied by many researchers (e.g., the authors of [6, 22, 24, 25] applied it to study bubble evolutions).

The governing equations in the model are solved using the finite volume method through the commercial software Ansys Fluent [14]. A coupled pressure-based algorithm has been employed in solving the equations and the discretization of time is performed through a firstorder implicit method [14]. The solution procedure is as follows [14]:

(1) The two momentum equations and the continuity equation are first simultaneously solved by employing a second-order upwind scheme, based on which the velocities, $v_{r}$ and $v_{z}$, and the pressure $p$, can be obtained. 
(2) Then the energy equation is solved using a second-order upwind scheme to get the temperature, $T$. The equation in the VOF method that governs the phase volume fractions in each numerical control volume is also solved to get the liquid and vapor fractions in every numerical control volume, based on which the liquid-vapor interface (i.e., the bubble wall) can be obtained.

(3) The density is obtained based on the corresponding equation of state (EOS) for each phase (the ideal gas EOS for water vapor and the Tait EOS, Eq.(4), for liquid water).

(4) The above solution procedure will be iterated until relevant convergence criteria are met, and then all the variables, $\left(\rho, p, v_{r}, v_{z}, T\right)$, and the bubble wall location are obtained for the current time step, and the solution process can go to the next numerical time step to repeat the same procedure.

The major material properties used in the simulations are taken from the literature [7-9, 14-15], and some of them have been listed in Table 1 of this paper.

\section{Results and Discussions}

First, the developed model will be verified by comparing its predictions with experimental measurements in the literature for bubble evolutions near a solid surface without a microhole sidewall confinement [26]. Figure 3 shows the comparison of the predictions from this model with the experimental observations in [26] for bubble evolutions near a solid wall. The model calculation starts at the moment when the bubble reaches its maximum radius of $\mathrm{R}_{\max }$ ( $\mathrm{R}_{\max }$ is around $1.45 \mathrm{~mm}$ based on the information from [26] for the experimental results in Fig.3), and this moment is defined as $\mathrm{t}=0$ in the model calculation. 
In the experiments in [26], the size of the solid specimen below the bubble is much larger than the bubble maximum radius of $1.45 \mathrm{~mm}$. Hence, it should be reasonable to assume that the exact specimen size in the $\mathrm{r}$ direction and the boundaries of the water container do not significantly affect the bubble evolution. Hence, in the model calculation the computational domain in Fig. 1a is used, where the values of $r_{\max }$ and $z_{\max }$ are chosen to be sufficiently large such that the shock wave due to bubble evolutions does not reach the boundaries of $\mathrm{z}=\mathrm{Z}_{\max }$ or $\mathrm{r}$ $=r_{\max }$ during the simulated period. Based on the information and images given in [26], it should be reasonable to assume the solid surface is a flat surface in the model calculations.

The initial conditions of the bubble at $\mathrm{t}=0$ assumed in the model calculation are shown in Fig. 2. In [26], the bubble is generated by focusing a pulsed laser beam into water at a point above a solid specimen. Hence, it is assumed in the model calculation that the bubble is filled with water vapor. For the experimental results given in Fig. 3 (taken from [26]), the bubble center at the moment of its formation is $\sim 2.32 \mathrm{~mm}$ away from the solid surface based on the information given in [26]. Hence, it is assumed that the bubble center is also $2.32 \mathrm{~mm}$ away from the solid surface at $t=0$ in the model calculation. Based on the experimental condition description in [26], it should be reasonable to assume that the experiment in [26] was performed in a common ambient environment at a common room temperature. Hence, in the model calculation, it is assumed that the initial temperature for the liquid water and for the water vapor inside the bubble is $295 \mathrm{~K}$ at $\mathrm{t}=0$. Following Ref. [11], it is assumed in the model calculation that the pressure of the vapor inside the bubble at $t=0$ is equal to the saturation pressure at the initial temperature (at $295 \mathrm{~K}$, the water vapor saturation pressure is around $2671 \mathrm{~Pa} \mathrm{[15]).}$

Based on the above initial conditions at $\mathrm{t}=0$, the model can then predict the subsequent bubble evolution. Figure 3 top row shows the experimentally-captured images of the bubble 
during the last few microseconds before its collapse in the experiment (taken from [26]). Figure 3 bottom row shows the model-predicted bubble size and shape evolution during the last few microseconds before the bubble collapse time in the model calculation. It can be seen that the model-predicted bubble size and shape evolutions agree well with the experimental observations given in the figure.

Next, the experimentally tested model will be used to study the effect of microhole sidewall on the bubble evolution and the bubble-generated shock waves. The simulation results are shown in Figs. 5 to 9 , for which the assumed bubble initial conditions at $\mathrm{t}=0$ in the calculations are given in Fig. 4 (and the assumed liquid water initial conditions and the domain boundary conditions are given in Fig.1). The model calculations start at $t=0$ when a highpressure small bubble is assumed to pre-exist, and at $\mathrm{t}=0$, it is assumed that the bubble has a radius of $2.64 \mu \mathrm{m}$, and is filled with water vapor that has a temperature of $1650 \mathrm{~K}$ and a pressure of $110 \mathrm{MPa}$. At $\mathrm{t}=0$, the bubble center is $200 \mu \mathrm{m}$ away from the solid surface below the bubble.

Figure 5 shows the model-predicted pressure history at the center of the solid wall surface ( $\mathrm{r}=0$ and $\mathrm{z}=0$ ) below the bubble, under three situations: (a) the bubble is located above a solid flat surface without a microhole sidewall confinement, (b) the bubble is inside a microhole with a diameter of $\mathrm{D}=400 \mu \mathrm{m}$ and a depth of $\mathrm{H}=400 \mu \mathrm{m}$, and (c) the bubble is inside a microhole with a diameter of $\mathrm{D}=200 \mu \mathrm{m}$ and a depth of $\mathrm{H}=400 \mu \mathrm{m}$. The pressure value is normalized by $\mathrm{P}_{\mathrm{mf}}$, which is the peak pressure for the situation without the hole sidewall confinement (Situation a). Situation (a), (b) and (c) corresponds to the pressure history curve (a), (b) and (c) in Fig. 5, respectively.

Figure 5 shows that when there is no sidewall confinement (Situation (a)), the pressure will remain at its initial value for some time, and then at around $t=\sim 130 \mathrm{~ns}$, the pressure starts 
increasing and reaches the peak value at $\mathrm{t}=\sim 139 \mathrm{~ns}$, and then the pressure decreases. However, when there is a microhole sidewall confinement, the pressure history becomes very different. When the hole diameter $\mathrm{D}$ is equal to $200 \mu \mathrm{m}$ (Situation $(\mathrm{c})$, where $\mathrm{H} / \mathrm{D}=2$ ), the pressure history before $\mathrm{t}=\sim 182 \mathrm{~ns}$ is very similar to Situation (a): the pressure reaches the first peak value and then decreases. However, the pressure increases again at $\mathrm{t}=\sim 182 \mathrm{~ns}$, and then at $\mathrm{t}=\sim 195 \mathrm{~ns}$ reaches a second peak value that is more than 7 times the first peak pressure value, and then the pressure quickly decreases. For the situation where $\mathrm{D}=400 \mu \mathrm{m}$ (Situation $(\mathrm{b})$, where $\mathrm{H} / \mathrm{D}=1)$, the pressure history before $\mathrm{t}=\sim 295 \mathrm{~ns}$ is also very similar to Situation (a). However, the pressure increases again at $\mathrm{t}=\sim 295 \mathrm{~ns}$, and then reaches a second peak value that is over five times the first peak pressure value.

It should be noted that in Figure 5, the pressure history curves for Situation (a) and (c) almost overlap with each other until $\mathrm{t}=\sim 182 \mathrm{~ns}$, and the curves for Situation (a) and (b) almost overlap with each other until $t=\sim 295$ ns. Hence, Situations (a), (b) and (c) have the same first pressure peak at $t=\sim 139$ ns, which is the only peak for Situation (a). Situation (c) has the second peak at $\mathrm{t}=\sim 195 \mathrm{~ns}$, while Situation (b) has the second peak at $\mathrm{t}=\sim 308 \mathrm{~ns}$.

The pressure histories in Fig. 5 can be understood based on the 2D pressure contour plots at different times shown in Fig. 6 and Fig. 7, for the situations without and with the hole sidewall confinement, respectively. Figure 6 shows that when there is no hole sidewall confinement, starting from $t=0$, the high-pressure small bubble starts growing, and pushes the ambient liquid water, and sends a shock wave into the ambient water. Before the shock wave reaches the solid surface below the bubble, the solid surface pressure will remain close to its initial pressure. However, at around $\mathrm{t}=\sim 130 \mathrm{~ns}$, the shock wave front reaches the point of $\mathrm{r}=0$ on the solid wall surface, where the pressure starts increasing, reaches the peak value and then decreases with 
time. This has explained the pressure history in Fig. 5 for the situation without the hole sidewall confinement (Situation (a)).

For the situation with the microhole sidewall confinement, Figure 7 shows that, similar to Fig. 6, the shock wave due to the bubble growth reaches the hole bottom wall surface at around $t$ $=\sim 130 \mathrm{~ns}$ (see Fig. $7 \mathrm{~F}$ ), when the pressure at the center of the hole bottom wall surface will start increasing, and then reach a peak value, and then decrease.

However, Figure 7 also shows that the shock waves due to the bubble growth will also propagate towards the hole sidewall. The shock waves reach the sidewall, and then get reflected by the wall, and the reflected waves collide with the bubble and form two high-pressure regions: one above the bubble and one below the bubble. The high-pressure region below the bubble propagates downwards and hits the hole bottom. This will cause the second pressure peak at the hole bottom as shown in Fig. 5 (for Situation (c)).

To be more specific, the shock wave reaches the sidewall at around $\mathrm{t}=\sim 64 \mathrm{~ns}$ as shown in Fig. 7C, and is then reflected by the sidewall. In the 2D r-z plane as shown in Fig. 7, the shock waves reflected by the left sidewall and by the right sidewall will collide with the bubble soon after around $t=\sim 120 \mathrm{~ns}$ as shown in Fig.7E and 7F (in a 3D domain, if one observes in $-\mathrm{Z}$ direction from above the hole, one will see that the shock waves are reflected by the sidewall and then focus towards $r=0$ ). Before the collision, the contour plot in Fig. 7E shows a continuous high-pressure red region at each reflected shock wave front. After the collision, Figure 7F shows that each continuous red region is split into two separate red regions: an upper red region and a lower red region (hence totally there are two upper red regions and two lower red regions). By $\mathrm{t}$ $=\sim 140 \mathrm{~ns}$ as shown in Fig. 7H, the two upper and two lower red regions have coalesced into one upper and one lower red region, respectively. Due to the interactions among the bubble and the 
reflected shock waves (particularly the high pressure region coalition), the peak pressure in the entire domain has been increased from around 3.8 MPa in Fig.7E to around $13 \mathrm{MPa}$ in Fig. 7H. The lower high-pressure red region propagates towards the hole bottom wall surface as shown in Fig. $7 \mathrm{H}$ to $7 \mathrm{~L}$, and has already reached the bottom wall surface by $\mathrm{t}=\sim 195 \mathrm{~ns}$ (see Fig. $7 \mathrm{~L}$ ), which has led to the second pressure peak observed in Fig. 5 (for Situation (c)).

In summary, Figs. 5 and 7 show that the reflection of the shock waves by the microhole sidewall and the interactions among the reflected waves and the bubble have led to a second and much higher pressure peak at the center of the hole bottom wall surface. Under the studied conditions, the pressure enhancement is larger for a smaller hole diameter when the hole depth remains the same.

Due to the sidewall confinement effect, not only the center point, but also the other points of the hole bottom wall surface have an enhanced peak pressure, which can be seen in Fig. 8. The figure shows the peak pressures at different locations of the solid wall surface below the bubble (it should be noted that the peak pressures at different locations may occur at different times). It can be seen that for $\mathrm{D}=200 \mu \mathrm{m}$ the peak pressures at all the given points in the figure are higher than those at the corresponding points for $\mathrm{D}=400 \mu \mathrm{m}$, which are higher than those without the hole sidewall confinement. The figure also shows that the peak pressures are relatively uniform at different locations shown in the figure when there is no microhole sidewall confinement. However, when the sidewall confinement exists, the peak pressures at the center ( $\mathrm{r}$ $=0)$ are much higher.

Figure 9 shows the bubble radius evolution with time, where the radius value is normalized by the bubble's initial radius at $\mathrm{t}=0$. It can be seen that before around $\mathrm{t}=\sim 135 \mathrm{~ns}$ the bubble radius with the hole sidewall confinement is very close to that without the hole 
sidewall confinement. However, after around $\mathrm{t}=\sim 135 \mathrm{~ns}$, the former becomes smaller due to the effect of the shock waves reflected by the sidewall.

The model used in this study has shown a reasonable accuracy in the comparison with the experimental measurements shown in Fig.3. It is expected that the model accuracy is sufficient for the major purpose of this paper, which is to qualitatively or semi-quantitatively reveal the effect of the microhole sidewall confinement on the water bubble evolution and the bubblegenerated shock waves. However, there is certainly still a lot of room to further improve the model, such as the consideration of the possible mass transfer across the bubble wall due to the vaporization or condensation process if occurs (which has been neglected in the model in this paper, and also in some other researchers' work such as Ref. [6]), and the enhancement of the accuracy of bubble initial conditions and material properties used in the model calculations.

Finally, it should be noted that for Figs. 5 to 9, it is assumed that the bubble is filled with water vapor at $t=0$. It has been found that if the bubble is assumed to be filled with air that has similar initial conditions at $\mathrm{t}=0$, model calculation results similar to Fig. 5 can still be obtained. Hence, the exact content of the bubble (water vapor or air) does not significantly affect the major conclusion of this paper under the investigated conditions.

\section{Conclusions}

An experimentally verified model has been applied to study the effect of the microhole sidewall confinement on the water bubble evolution and the bubble-generated shock waves. Under the investigated conditions, it has been found that:

(1) When there is no microhole sidewall confinement, as the high-pressure small bubble grows, it sends a shock wave into the ambient liquid water. When the shock wave reaches the solid 
flat surface below the bubble, a pressure peak is generated at the center $(r=0)$ of the solid surface.

(2) When there is a microhole sidewall confinement, due to the reflection of shock waves by the sidewall and the interactions among the bubble and the reflected waves, a second and much higher pressure peak is generated at the center of the solid surface below the bubble (that is, the hole bottom wall surface).

(3) Due to the microhole sidewall confinement, besides the center point, the peak pressures at other points of the hole bottom wall surface have also been enhanced.

(4) The bubble radius under the microhole sidewall confinement is similar to that without the sidewall confinement during the early stage of the bubble growth, but becomes smaller in the later stage.

It is good work in the future to study the implications of the above discoveries on the related manufacturing processes and other applications that may involve water bubble evolutions, such as ultrasonic cleaning, cavitation peening, and laser-material interactions in water. It is also good work in the future to study how to utilize the above discoveries, particularly the revealed pressure enhancement effect due to the microhole sidewall confinement, to improve or benefit the related applications and manufacturing processes.

\section{Acknowledgement}

This material is based upon work supported by the National Science Foundation under Grant No. CMMI 1055805 and CMMI 1543865. The authors appreciate the help by Dr. Hamid Arastoopour at Illinois Institute of Technology related to the use of Ansys Fluent. 


\section{References}

1. M. Qin, D.Y. Ju, and R. Oba, 2006, "Investigation of the influence of incidence angle on the process capability of water cavitation peening”, Surface \& Coatings Technology, 201, 1409-1413, 2006.

2. B. Niemczewski, "Observations of water cavitation intensity under practical ultrasonic cleaning conditions”, Ultrasonics Sonochemistry, 14, 13-18, 2007.

3. J. Cornel, "A closer look at acoustic cavitation", Report in Eindhoven University of Technology Chemical Engineering and Chemistry Process Development Group, 2005.

4. E. Samiei, M. Shams, and R. Ebrahimi, "A novel numerical scheme for the investigation of surface tension effects on growth and collapse stages of cavitation bubbles", European Journal of Mechanics B/Fluids, 30, 41-50, 2011.

5. S. Popinet, and S. Zaleski, "Bubble collapse near a solid boundary: a numerical study of the influence of viscosity", J. Fluid Mech., 464, 137-163, 2002.

6. R.M.B. Teymouri, and G. Ahmadi, "Intensification of the near wall collapsing bubble induced jet using an opposite secondary wall", Journal of Fluid Science and Technology, 3, 207-218, 2008.

7. E.A. Neppiras, “Acoustic cavitation”, Physics Reports, 61(3), 159-251, 1980.

8. F.R. Gilmore, "The growth or collapse of a spherical bubble in a viscous compressible liquid", Report No. 26-4, Hydrodynamics Laboratory, California Institute of Technology, Pasadena, California, USA, 1952.

9. W. Lauterborn and T. Kurz, "Physics of bubble oscillations", Reports on Progress in Physics, 73, 106501, 2010.

10. E.A. Brujan and Y. Matsumoto, "Collapse of micrometer-sized cavitation bubbles near a rigid boundary", Microfluid Nanofluid, 13, 957-966, 2012.

11. I. Akhatov, O. Lindau, A. Topolnikov, R. Mettin, N. Vakhitova, and W. Lauterborn, "Collapse and rebound of a laser-induced cavitation bubble", Physics of Fluids, 13(10), 2805-2819, 2001.

12. T.G. Leighton, The Acoustic Bubble, Academic Press Limited, London, 1994.

13. J.C. Tannehill, D.A. Anderson, and R.H. Pletcher, Computational Fluid Mechanics and Heat Transfer, Taylor \& Francis, Washington DC, $2^{\text {nd }}$ edition, 1997.

14. Ansys, Inc., “Ansys FLUENT Theory Guide”, Release 13, 2010 (some material properties available in the Ansys-Fluent database are also used in the model calculations).

15. Y.A. Cengel, J.M. Cimbala, and R.H. Turner, Fundamentals of Thermal-Fluid Sciences, McGraw-Hill Higher Education, 4 ${ }^{\text {th }}$ edition, 2012.

16. C.W. Hirt, and B.D. Nichols, "Volume of fluid (VOF) method for the dynamics of free boundaries", Journal of Computational Physics, 39, 201-225, 1981.

17. R.L. Panton, Incompressible Flow, Wiley, Hoboken, NJ, $3^{\text {rd }}$ ed., 2005. 
18. S. Tao and B. Wu, "Nanosecond laser pulse interactions with breakdown plasma in gas medium confined in a microhole", Applied Physics B., 113, 251-258, 2013.

19. R.D. Sandberg, "Governing equations for a new compressible Navier-Stokes solver in general cylindrical coordinates", Report No. AFM-07/07, University of Southampton, School of Engineering Sciences, Aerodynamics \& Flight Mechanics Group, November 2007.

20. C.W. Hirt, and B.D. Nichols, "A computational method for free surface hydrodynamics", Journal of Pressure Vessel Technology, 103, 136-141, 1981.

21. W.J. Rider, D.B. Kothe, "Reconstructing volume tracking", Journal of Computational Physics, 141, 112-152, 1998.

22. M. van Sint Annaland, N.G. Deen, J.A.M. Kuipers, "Numerical simulation of gas bubbles behaviour using a three-dimensional volume of fluid method", Chemical Engineering Science, 60, 2999 - 3011, 2005.

23. J.U. Brackbill, D.B. Kothe, C. Zemach, “A continuum method for modeling surface tension”, Journal of Computational Physics, 100,335-354, 1992.

24. B.G.M. van Wachem, J.C. Schouten, "Experimental validation of 3-D lagrangian VOF model: bubble shape and rise velocity", AIChE Journal, 48(12), 2744-2753, 2002.

25. E. Delnoij, J.A.M. Kuipers, W.P.M. van Swaaij, "Numerical simulation of bubble coalescence using a volume of fluid (VOF) model”, Third International Conference on Multiphase Flow, Lyon, France, 1998.

26. A. Philipp, W. Lauterborn, "Cavitation erosion by single laser-produced bubbles", J. Fluid Mech. 361, 75-116, 1998. 


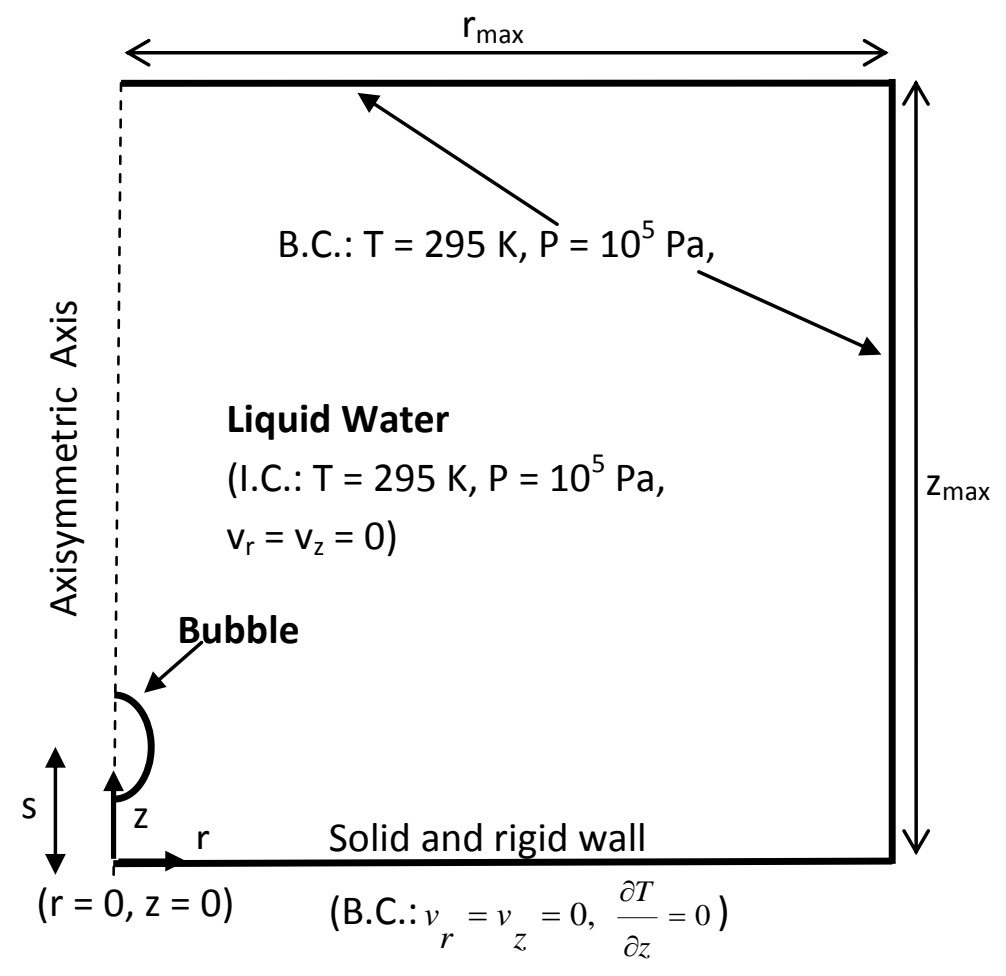

(a) 


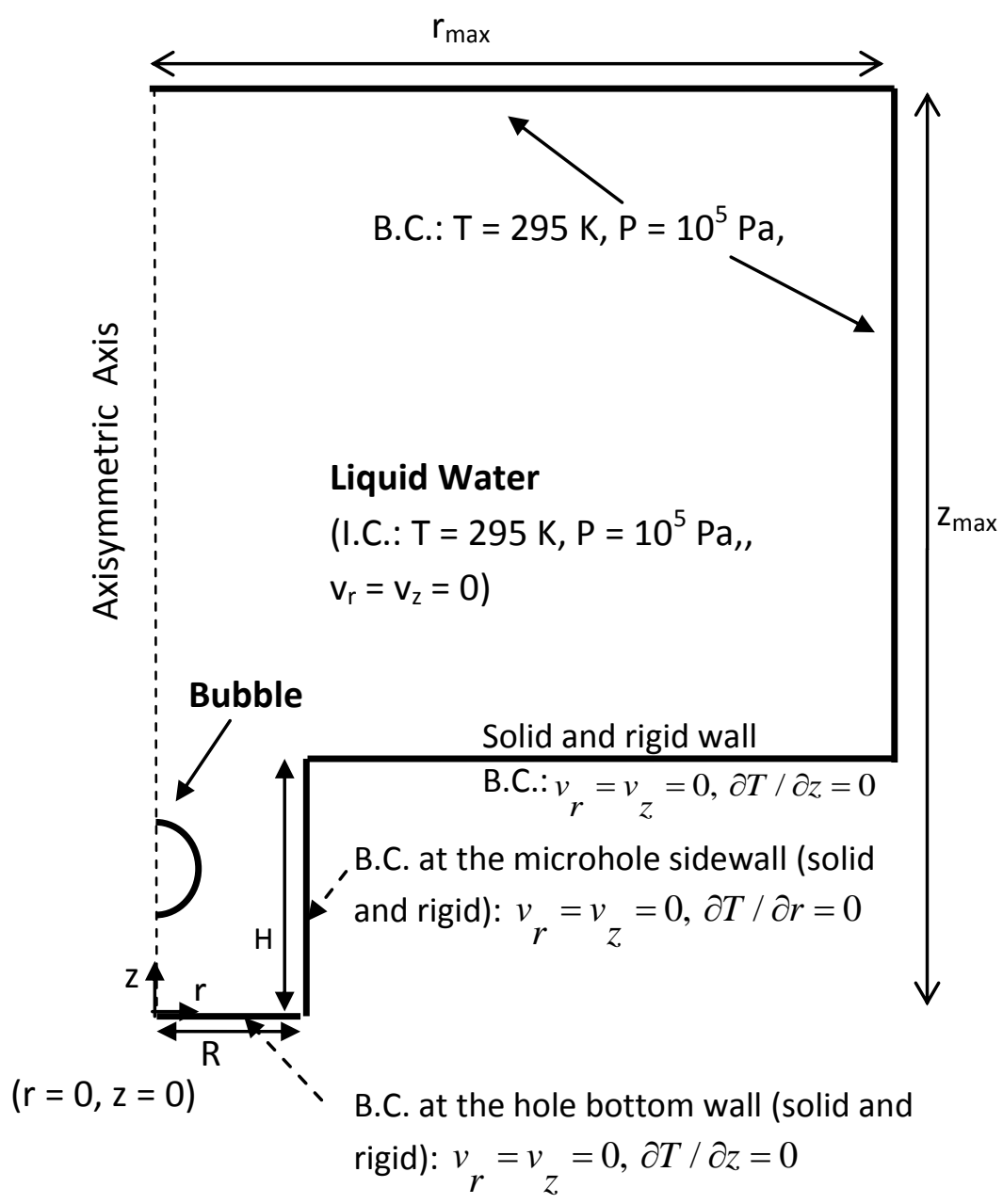

(b)

Figure 1. Schematic diagram of the model setup: (a) bubble evolution over a flat solid and rigid surface without a microhole sidewall confinement, and (b) bubble evolution inside a microhole with the sidewall confinement (hole radius: R, and depth: H; B.C.: boundary conditions; I.C.: initial conditions). This figure has shown the computational domain boundary conditions and the liquid water initial conditions, and see Figs. 2 and 4 for the assumed bubble initial conditions. 


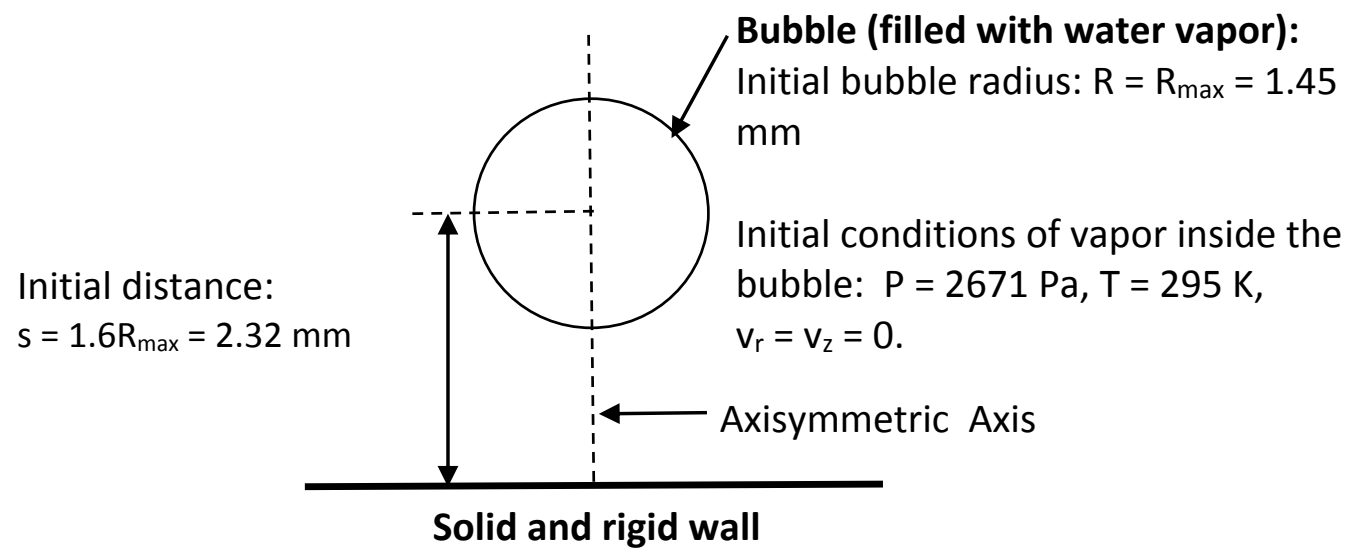

Figure 2. Schematic diagram showing the bubble initial conditions at $t=0$ assumed in the model calculations for the simulation results given in Fig. 3 (this figure has only shown a portion of the computational domain; please see Fig. 1a for the whole domain, the domain boundary conditions and the assumed liquid water initial conditions). 

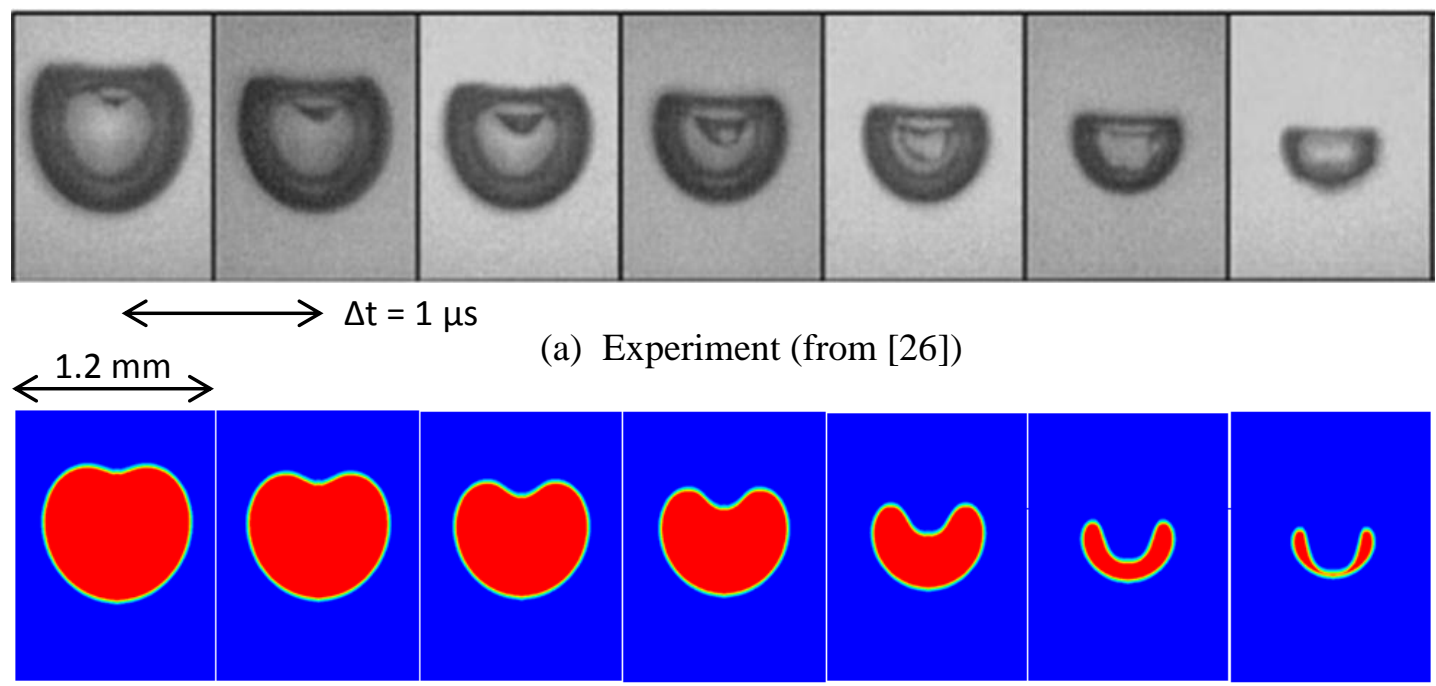

(b) Model prediction (from this paper)

Figure 3. (a) Experimentally captured images of a bubble evolving above a solid surface during the last few microseconds before its collapse in the experiment (images taken from Fig.3b of Ref. [26], (C) 1998 Cambridge University Press); and (b) The evolution of the shape of a bubble above a solid surface during the last few microseconds before its collapse in the model calculation, predicted by the model in this paper (the assumed initial bubble conditions in the model calculation are given in Fig. 2, and the assumed boundary conditions and the liquid water initial conditions are given in Fig.1a; the red color represents the bubble shape and the blue color denotes the surrounding water). For all the plots in (a) and (b), the width of each plot correspond to $1.2 \mathrm{~mm}$ in the physical domain, and each plot corresponds to a time that is $\sim 1 \mu \mathrm{s}$

after the plot on its immediate left side. The far right plot in (a) and (b) corresponds to the moment of $\sim 1 \mu$ s before the bubble collapses in the experiment and in the model calculation, respectively. 


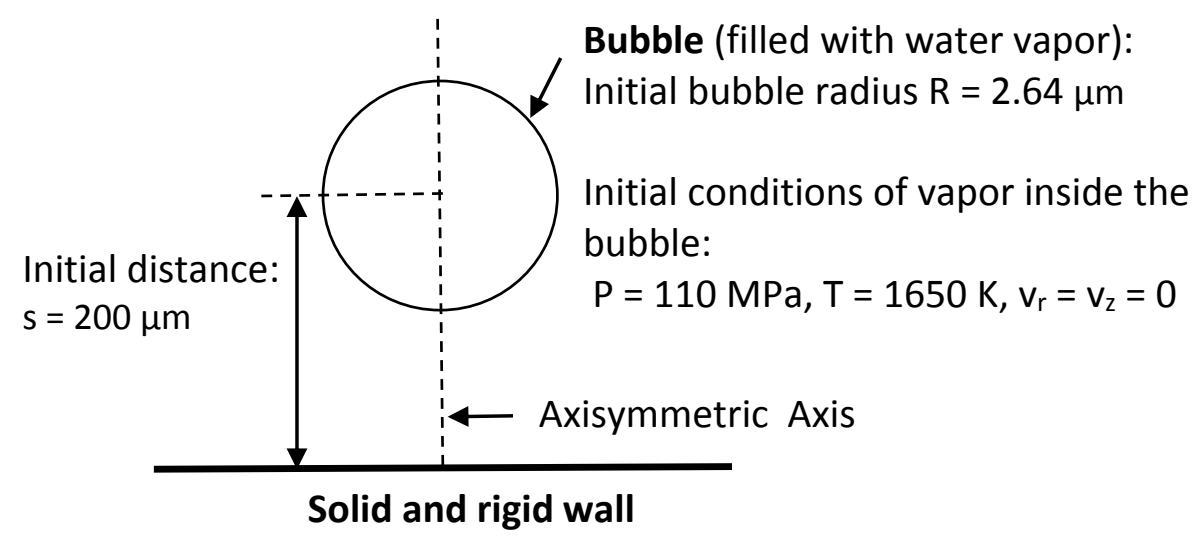

(a)

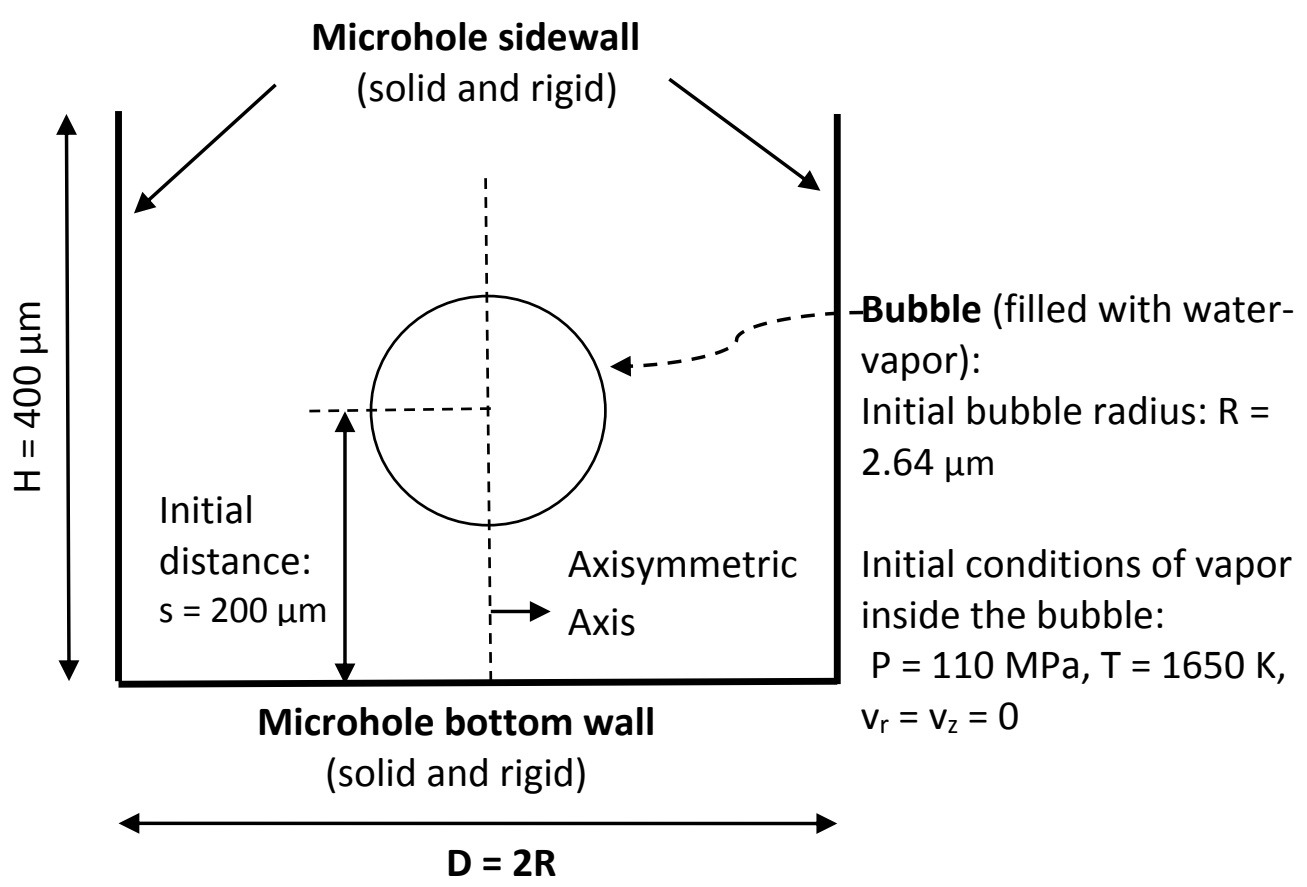

(b)

Figure 4. Schematic diagrams showing the assumed initial bubble conditions at $t=0$ for the model calculation results shown in Figs. 5 to 9, (a) without, and (b) with, microhole sidewall confinement (this figure has only shown a portion of the computational domain; please see Fig.1 $\mathrm{a}$ and $\mathrm{b}$ for the whole domain, the domain boundary conditions and the assumed liquid water initial conditions). 


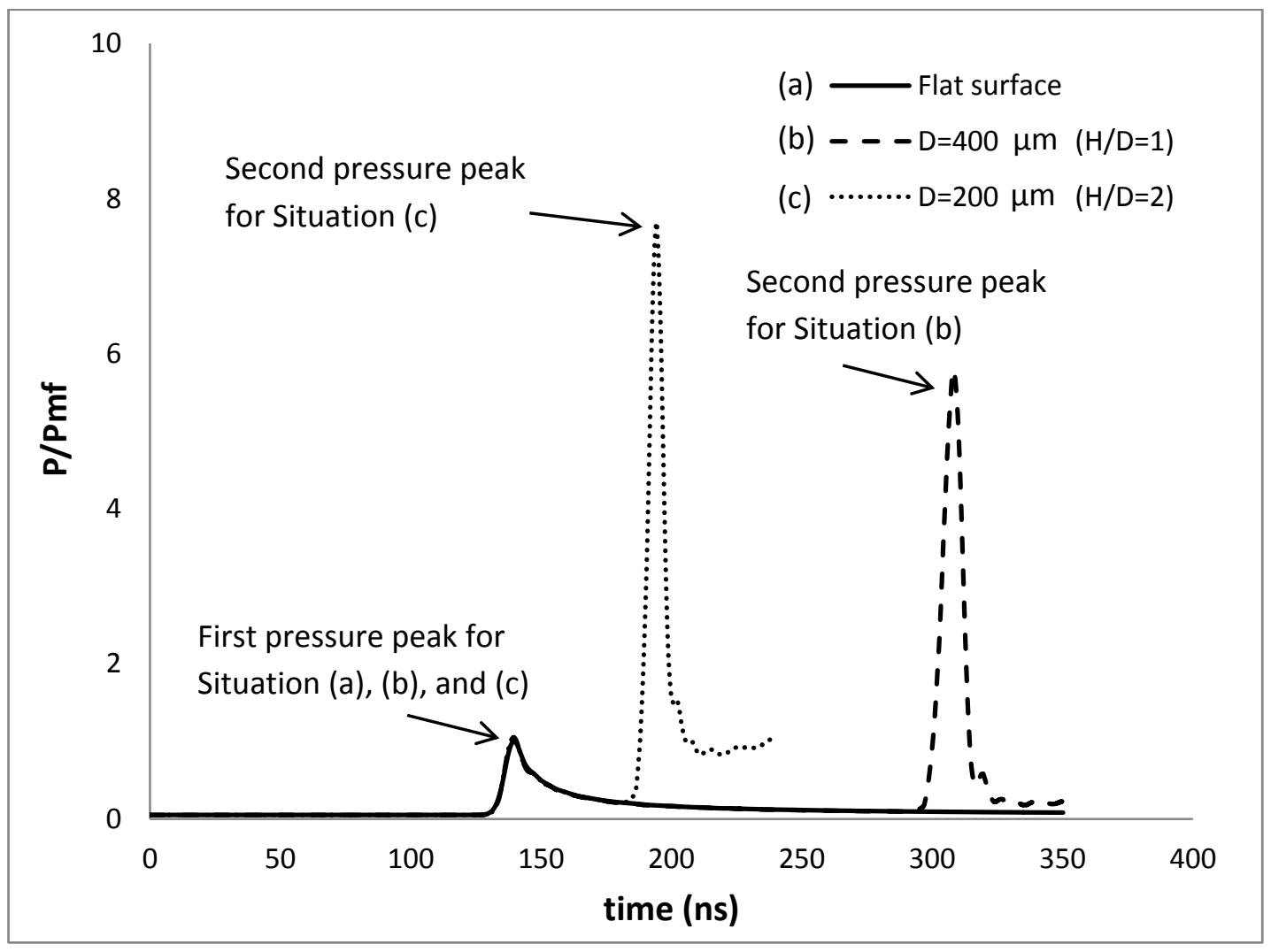

Figure 5. Model-predicted pressure history at the center of the solid wall surface $(\mathrm{r}=0, \mathrm{z}$ $=0$ ) below the bubble: (a) the bubble is above a flat surface without a microhole sidewall confinement, (b) the bubble is inside a microhole with a diameter of $\mathrm{D}=400 \mu \mathrm{m}$ and a depth of $\mathrm{H}=400 \mu \mathrm{m}$, and (c) the bubble is inside a microhole with a diameter of $\mathrm{D}=200 \mu \mathrm{m}$ and a depth of $\mathrm{H}=400 \mu \mathrm{m}$ (the pressures are normalized by $\mathrm{P}_{\mathrm{mf}}(=1.98 \mathrm{MPa})$, which is the peak pressure for situation (a)). The pressure history curves for Situation (a) and (c) almost overlap with each other until $\mathrm{t}=\sim 182 \mathrm{~ns}$, and the curves for Situation (a) and (b) almost overlap with each other until $\mathrm{t}=$ 295 ns. Hence, Situations (a), (b) and (c) have the same first pressure peak at $\mathrm{t}=\sim 139 \mathrm{~ns}$, which is the only peak for Situation (a). Situation (c) has the second peak at $t=\sim 195 \mathrm{~ns}$, while Situation

(b) has the second peak at $\mathrm{t}=\sim 308 \mathrm{~ns}$. Please see Fig. 4 for the bubble initial conditions assumed in the model calculations, and Fig.1 for boundary conditions and the liquid water initial conditions. 


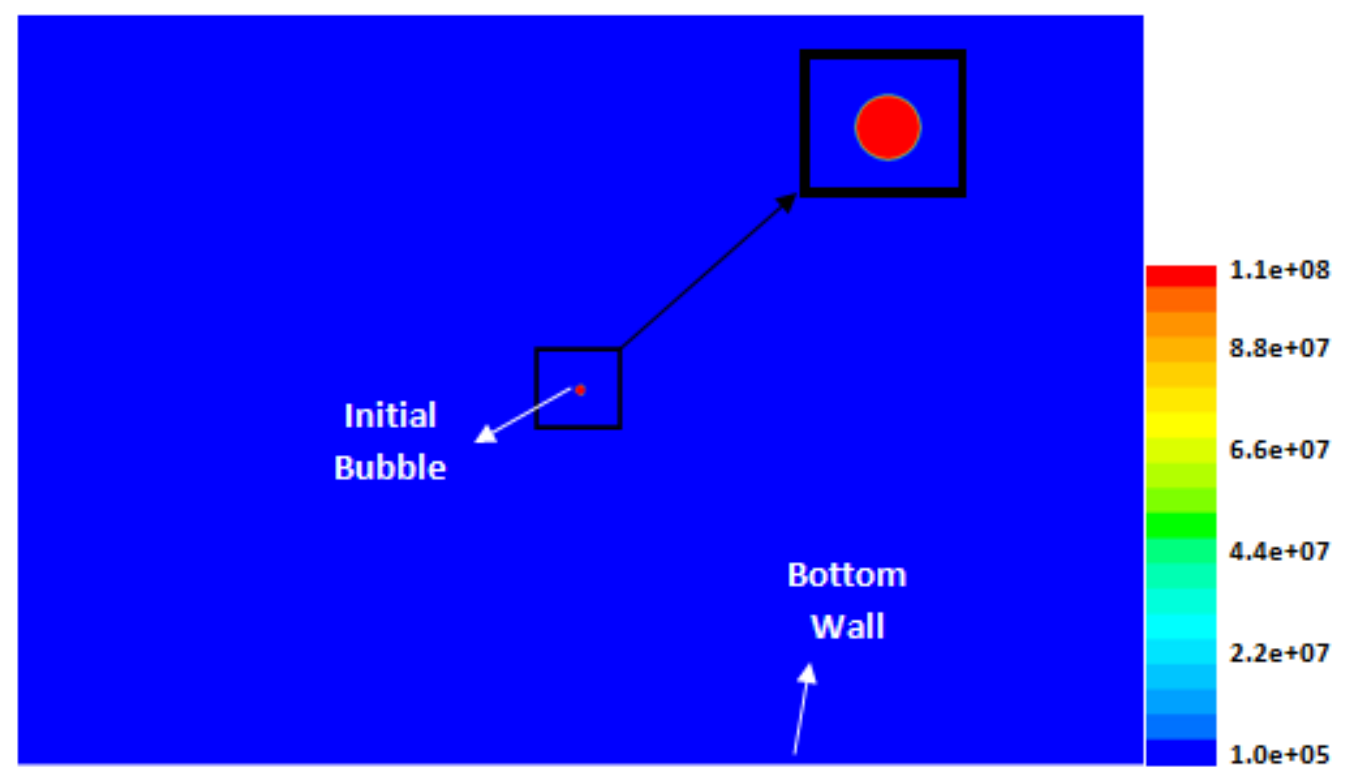

(a)

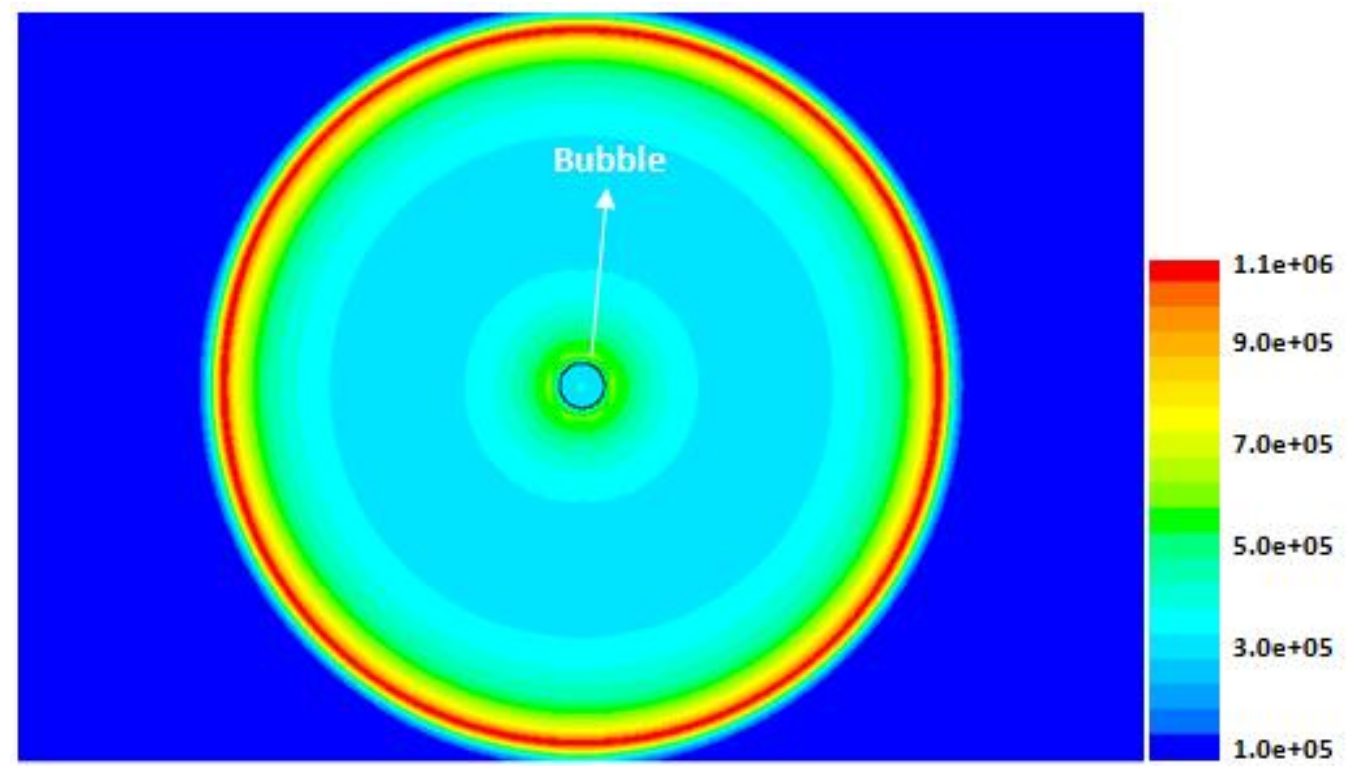

(b) 


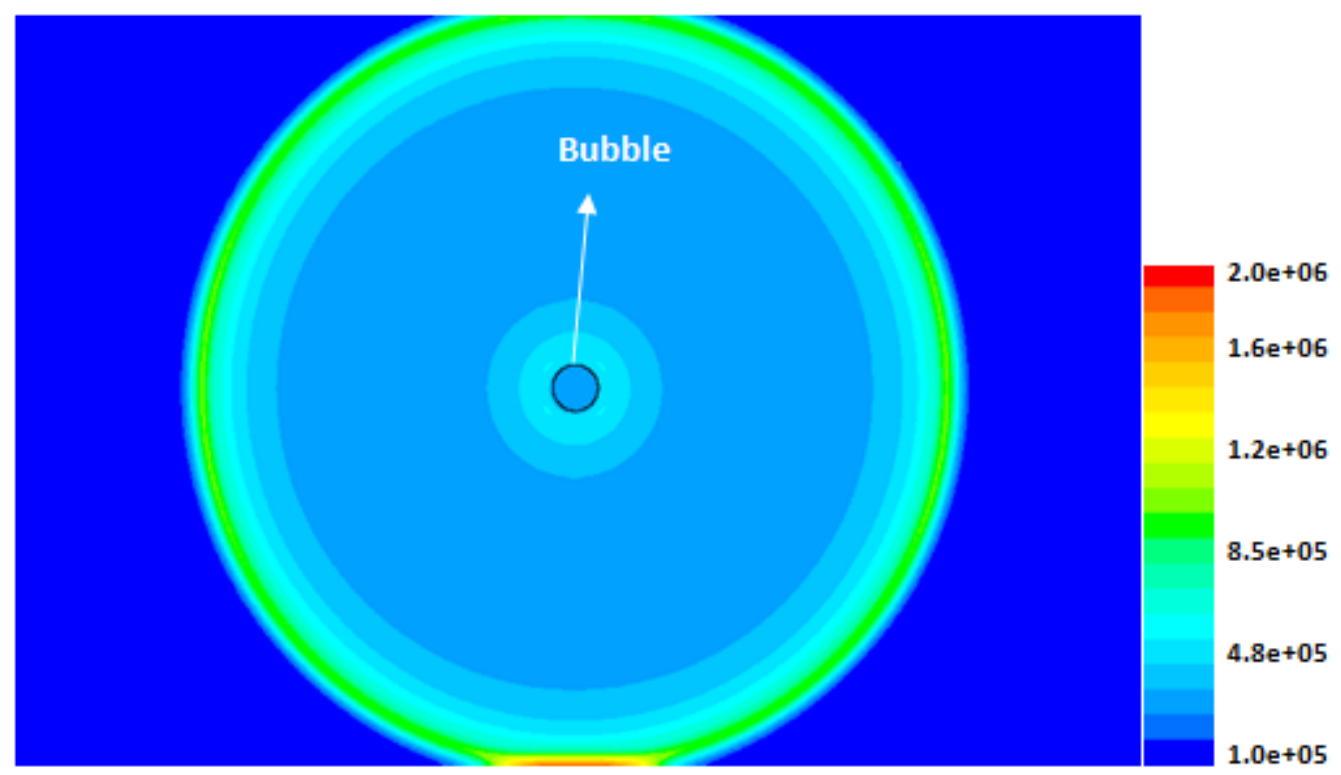

(c)

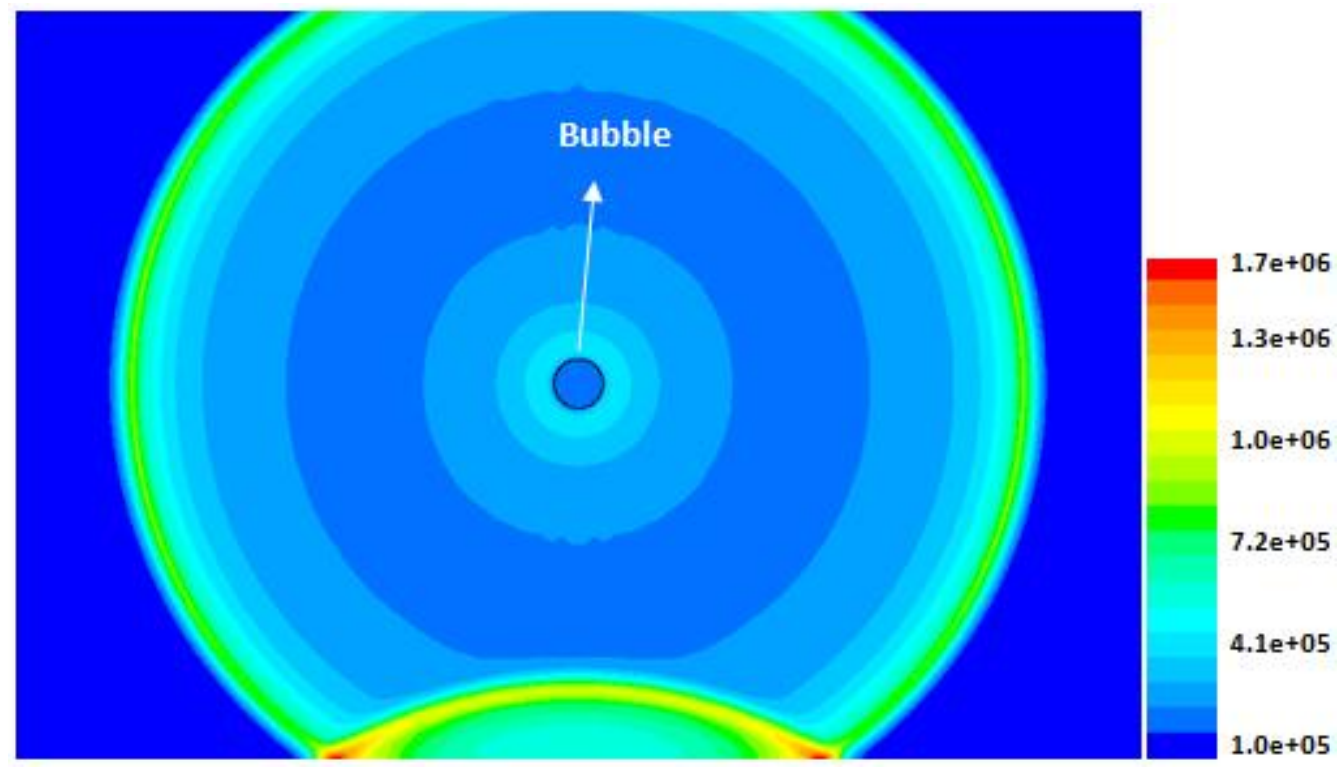

(d)

Figure 6. Model-predicted 2D pressure distributions for the bubble growth above a flat solid wall without a microhole sidewall confinement (the domain size is $600 \times 400 \mu \mathrm{m}$ for each plot): a) $\mathrm{t}=0$ $n s, b) t=132 \mathrm{~ns}, \mathrm{c}) \mathrm{t}=139 \mathrm{~ns}, \mathrm{~d}) \mathrm{t}=165 \mathrm{~ns}$. In each plot, the bubble boundary wall is indicated by a dark closed line, and the pressure unit is Pascal. Please see Fig. 4a for the bubble initial conditions assumed in the model calculations, and Fig.1a for boundary conditions and the liquid water initial conditions. 


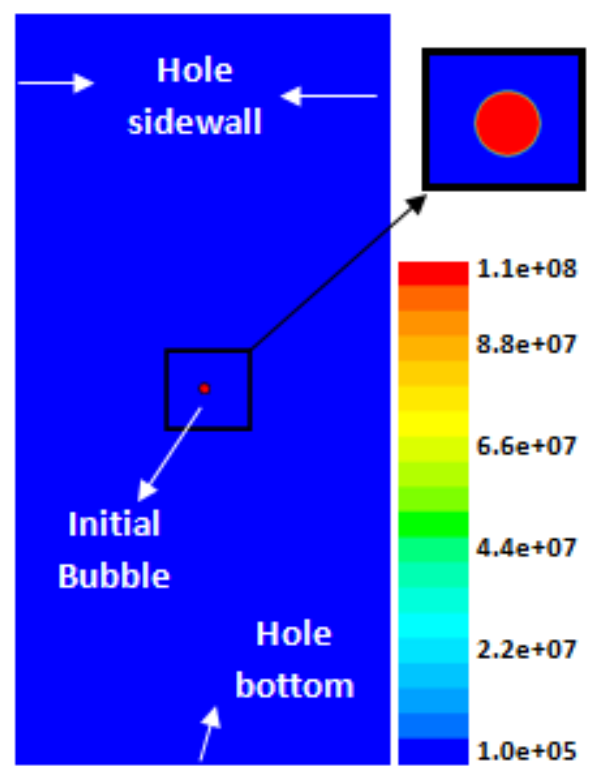

(A)

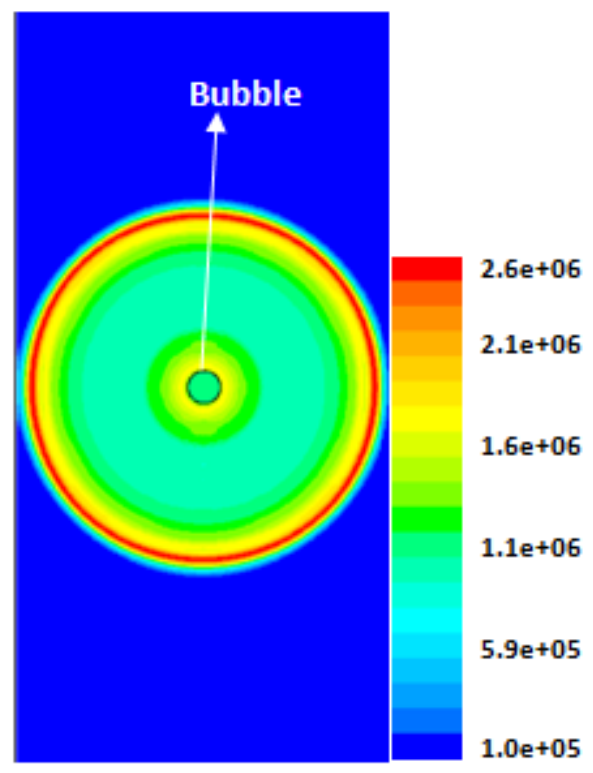

(C)

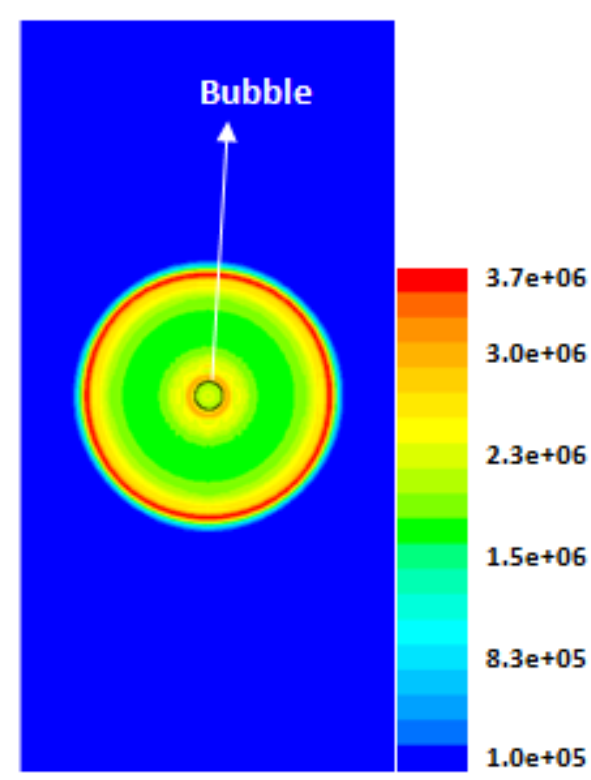

(B)

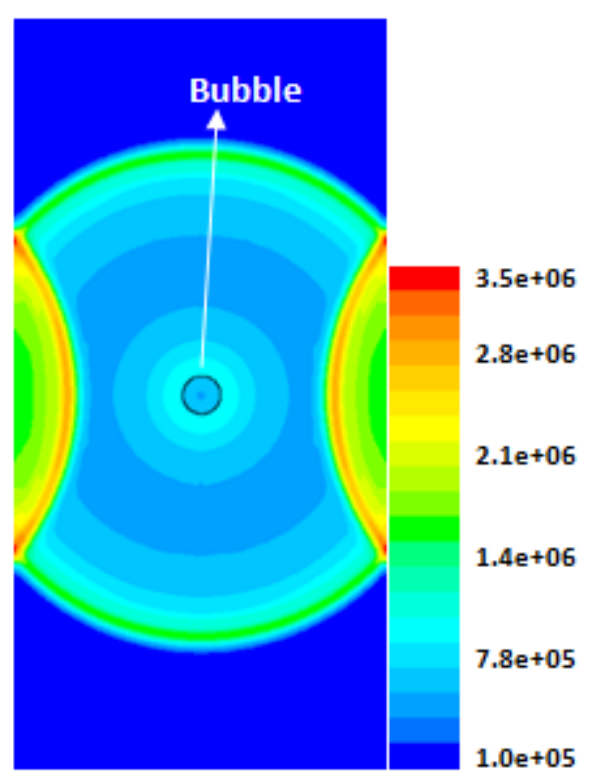

(D) 


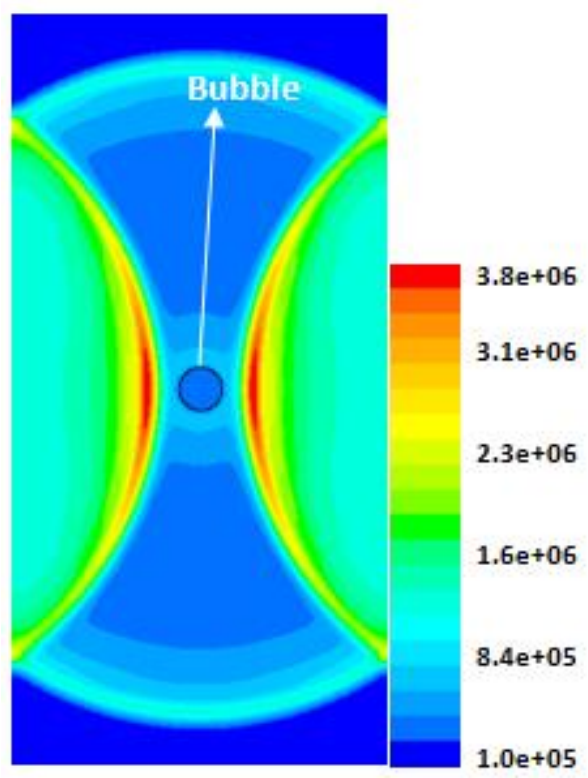

(E)

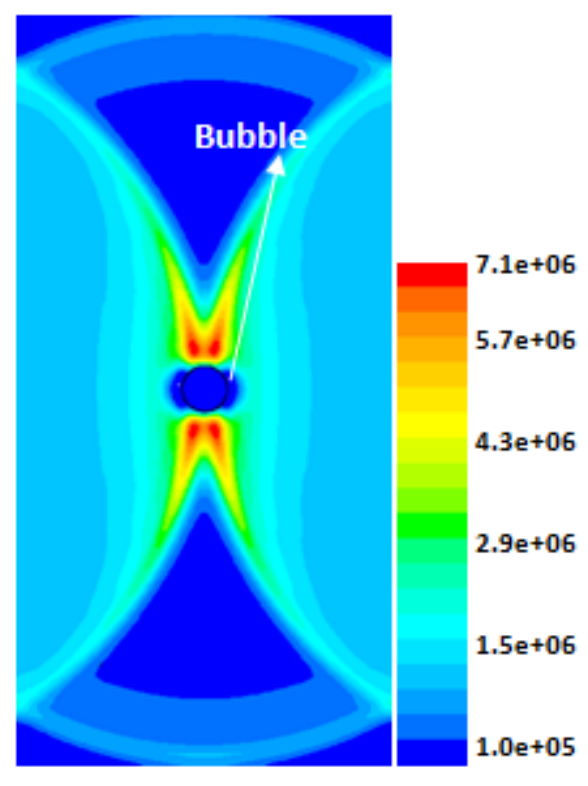

(G)

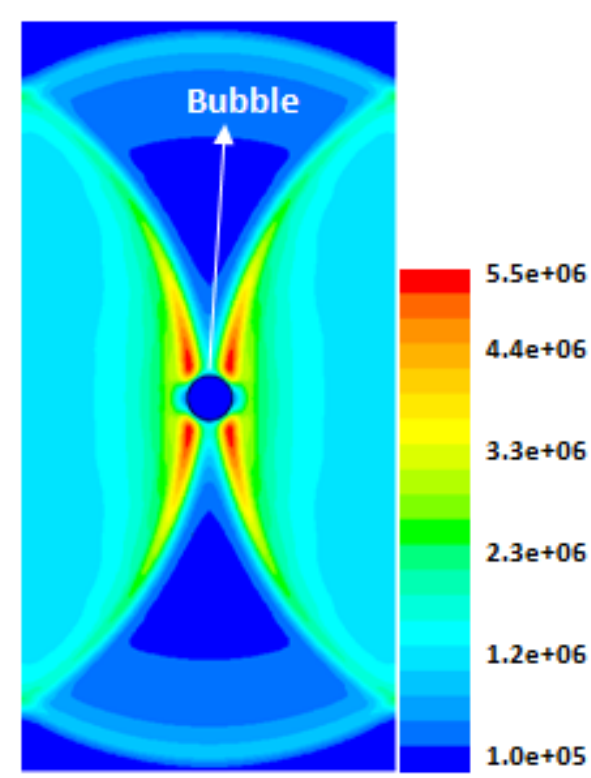

(F)

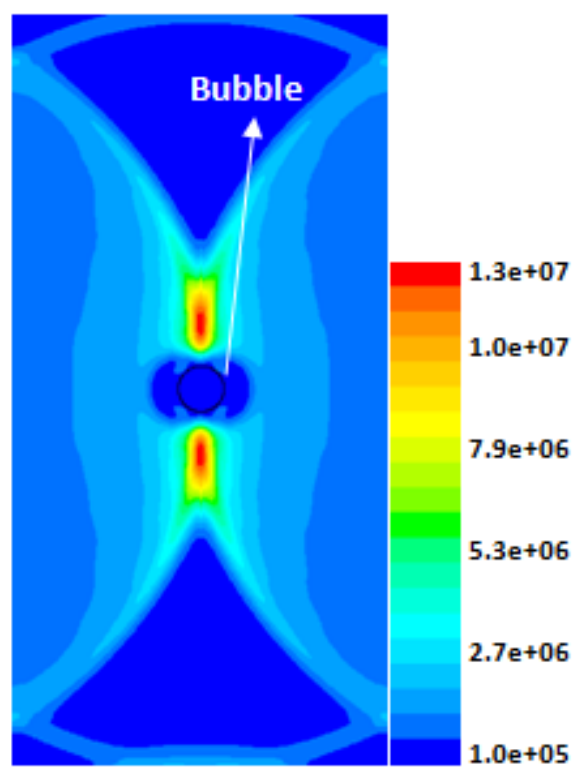

(H) 


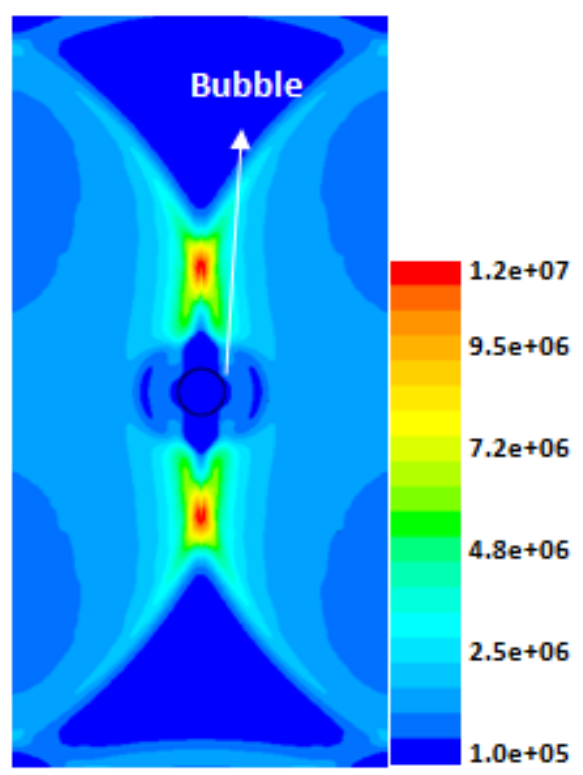

(I)

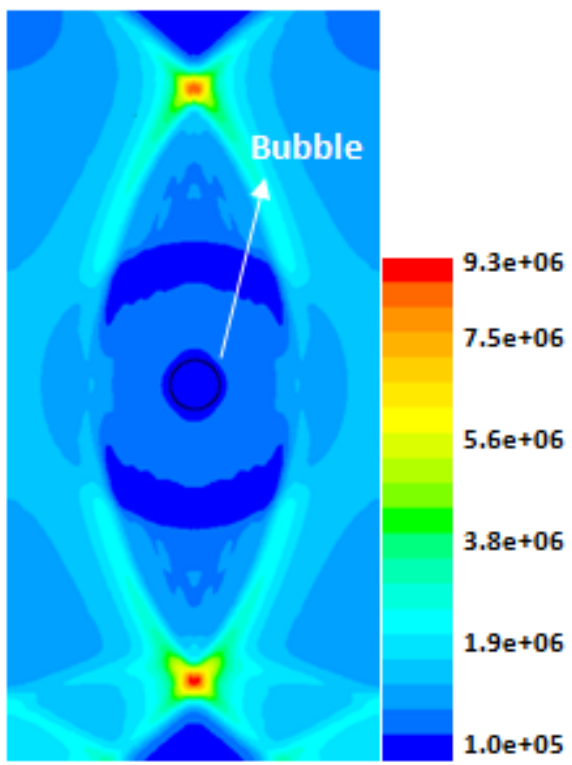

$(\mathrm{K})$

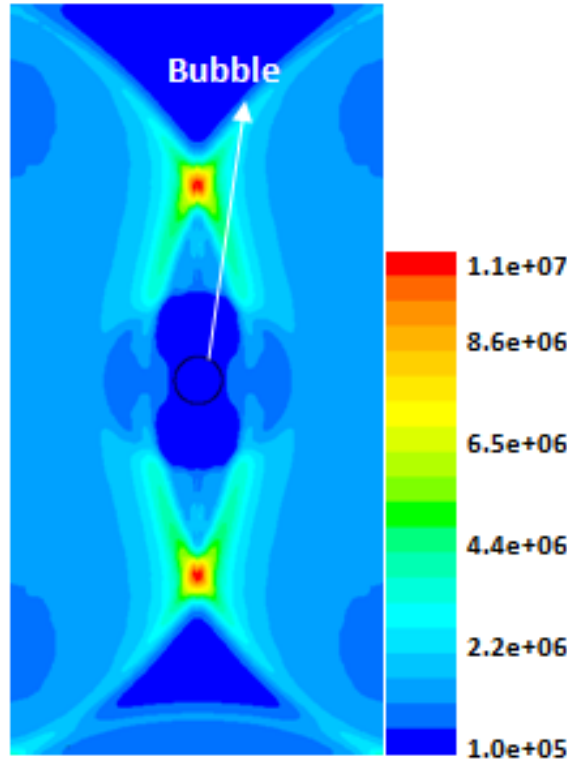

$(\mathrm{J})$

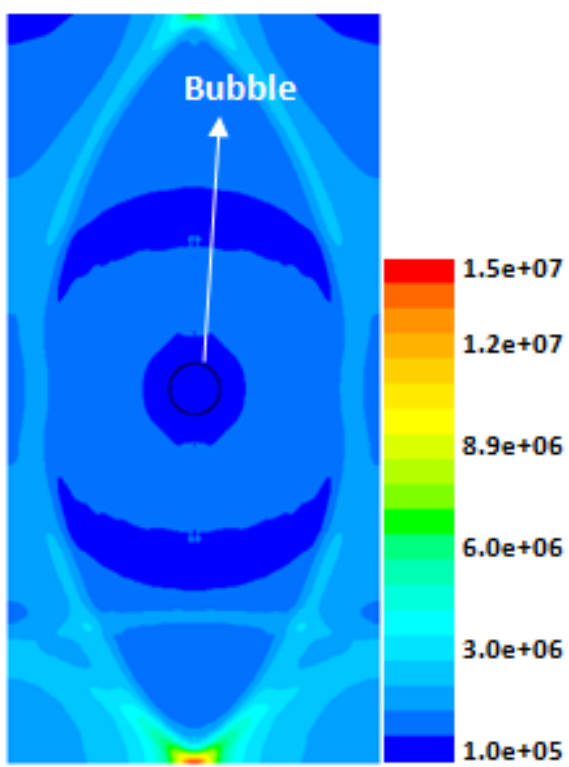

(L)

Figure 7. Model-predicted 2D pressure distributions for the bubble growth inside a microhole with a diameter of $\mathrm{D}=200 \mu \mathrm{m}$ and a depth of $\mathrm{H}=400 \mu \mathrm{m}$ (the domain size for each plot is 200 $\mu \mathrm{m} \times 400 \mu \mathrm{m}$ ): A) $\mathrm{t}=0 \mathrm{~ns}, \mathrm{~B}) \mathrm{t}=45 \mathrm{~ns}, \mathrm{C}) \mathrm{t}=64 \mathrm{~ns}$, D) $\mathrm{t}=90 \mathrm{~ns}, \mathrm{E}) \mathrm{t}=120 \mathrm{~ns}, \mathrm{~F}) \mathrm{t}=132 \mathrm{~ns}, \mathrm{G}) \mathrm{t}=135$

ns, H) $t=140$ ns, I) $t=145$ ns, J) $t=155$ ns, K) $t=175$ ns, L) $t=195$ ns. In each plot, the bubble boundary wall is indicated by a dark closed line. The pressure unit is Pascal. Please see Fig. 4b for the bubble initial conditions assumed in the model calculations, and Fig.1b for boundary conditions and the liquid water initial conditions. 


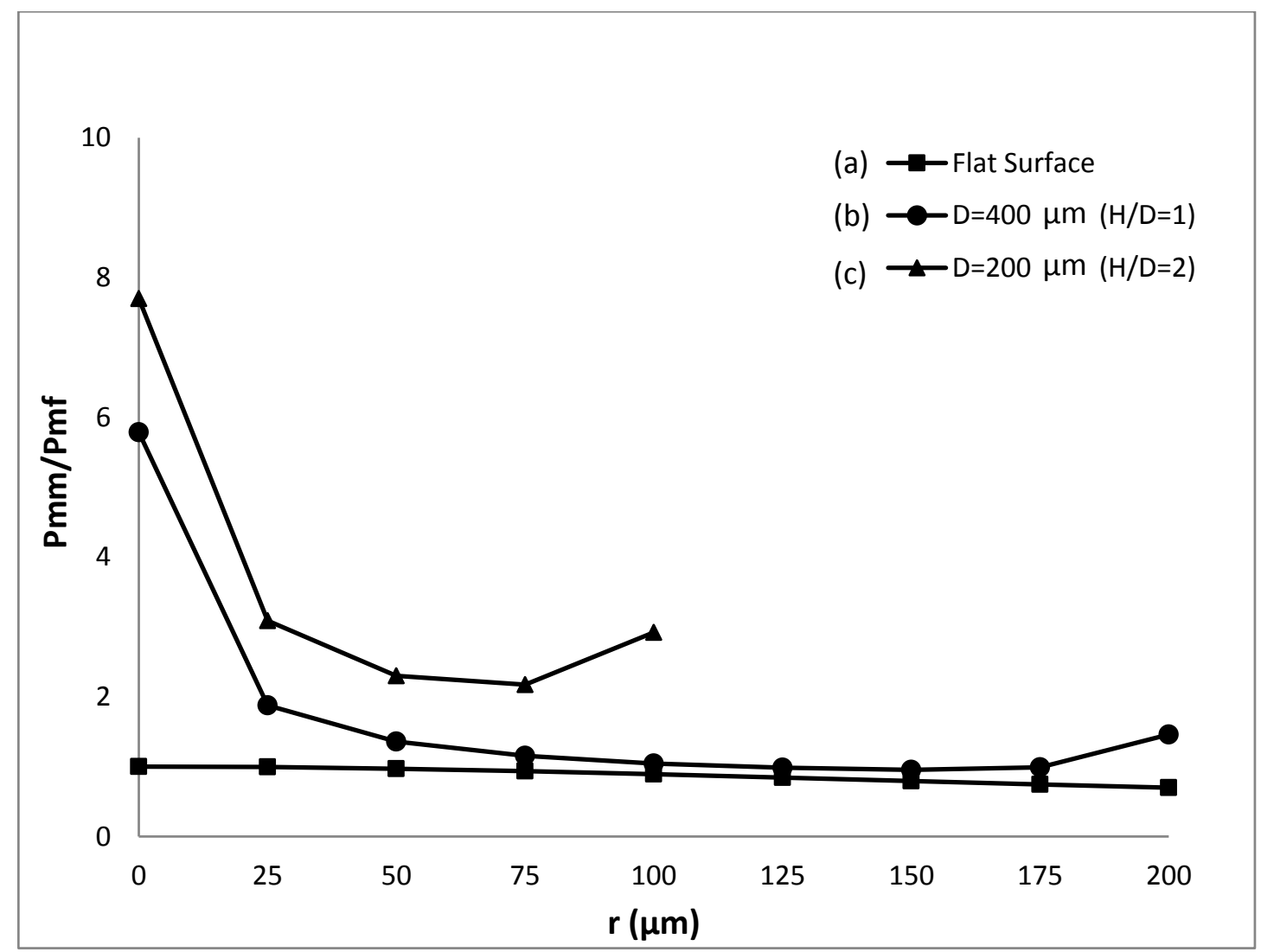

Figure 8. Model-predicted peak pressures, $\mathrm{P}_{\mathrm{mm}}$, at different locations of the solid wall surface below the bubble: (a) the bubble is above a flat solid wall surface without a microhole sidewall confinement, (b) the bubble is inside a microhole with a diameter of $\mathrm{D}=400 \mu \mathrm{m}$ and depth of $\mathrm{H}$ $=400 \mu \mathrm{m}$, and (c) the bubble is inside a microhole with a diameter of $\mathrm{D}=200 \mu \mathrm{m}$ and depth of $\mathrm{H}=400 \mu \mathrm{m}$ (the pressures are normalized by $\mathrm{P}_{\mathrm{mf}}(=1.98 \mathrm{MPa})$, which is the peak pressure at $\mathrm{r}=$

0 for situation (a)). Please see Fig. 4 for the bubble initial conditions assumed in the model calculations, and Fig. 1 for boundary conditions and the liquid water initial conditions. 


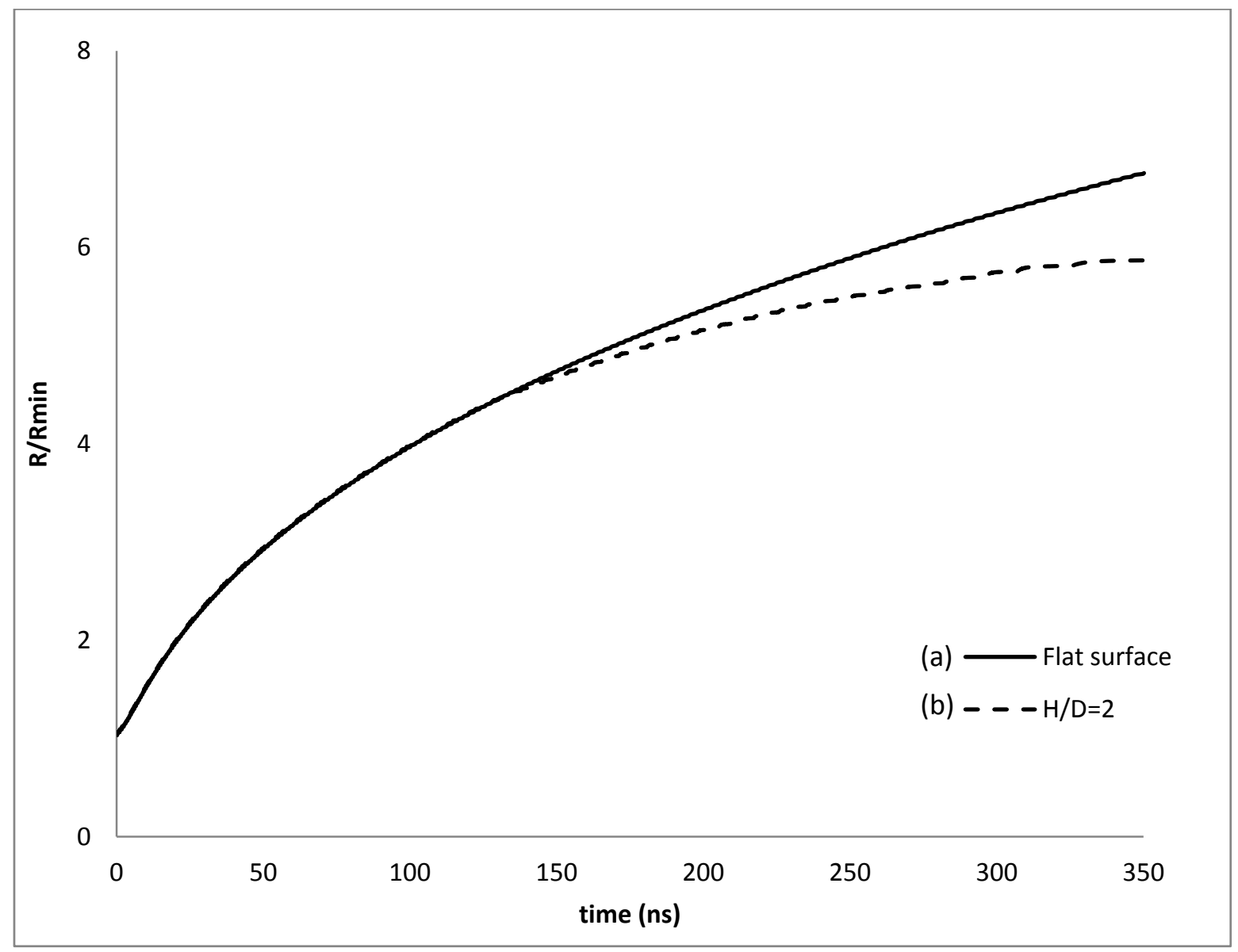

Figure 9. Model-predicted bubble radius evolution: (a) over a flat solid surface without a microhole sidewall confinement, and (b) inside a microhole with the microhole sidewall confinement $(\mathrm{D}=200 \mu \mathrm{m}$ and $\mathrm{H}=400 \mu \mathrm{m})$. The bubble radius, $R$, is normalized by its initial radius of $R_{\min }=2.64 \mu \mathrm{m}$. Please see Fig. 4 for the bubble initial conditions assumed in the model calculations, and Fig.1 for boundary conditions and the liquid water initial conditions. 
Table 1: Some of the Major Material Properties Used in the Model Calculations (The viscosity, thermal conductivity and specific heat of water vapor are considered to be temperature dependent in the model calculations, and are taken from the tabulated data in [15])

\begin{tabular}{|c|c|c|c|}
\hline & Description & value & Reference \\
\hline$\mu$ & Liquid water viscosity & $0.000958[\mathrm{~Pa} . \mathrm{s}]$ & {$[15]$} \\
\hline$\rho_{0}$ & Ambient liquid water density & $998\left[\mathrm{~kg} / \mathrm{m}^{3}\right]$ & {$[9,15]$} \\
\hline $\mathrm{K}$ & Liquid water thermal conductivity & $0.602[\mathrm{~W} / \mathrm{m} . \mathrm{K}]$ & {$[14-15]$} \\
\hline $\mathrm{C}_{\mathrm{p}}$ & Liquid water specific heat & $4182[\mathrm{~J} / \mathrm{kg} . \mathrm{K}]$ & {$[14-15]$} \\
\hline$\sigma$ & Water surface tension & $0.0725[\mathrm{~N} / \mathrm{m}]$ & {$[9]$} \\
\hline $\mathrm{B}$ & Parameter of Tait EOS (Eq.(4)) & $3000[\mathrm{bar}]$ & {$[7-8]$} \\
\hline $\mathrm{n}$ & Parameter of Tait EOS (Eq.(4)) & 7 & {$[7-8]$} \\
\hline $\mathrm{M}$ & Molar mass of water & $18.015[\mathrm{~kg} / \mathrm{kmol}]$ & {$[15]$} \\
\hline
\end{tabular}

